\title{
LBRP: A RESILIENT ENERGY HARVESTING NOISE Aware Routing Protocol For UNDER WATER SENSOR NETWORKS (UWSNS)
}

\author{
Md.Ashraful Islam ${ }^{1}$, Milon Biswas ${ }^{2}$, Md.Julkar Nayeen Mahi $^{3}$ and \\ Md.Whaiduzzaman ${ }^{4}$ \\ ${ }^{1,2}$ Lecturer, Department of Computer Science and Engineering, Bangladesh University \\ of Business and Technology (BUBT), Mirpur -2, Dhaka-1216, Bangladesh \\ ${ }^{3}$ Lecturer, Department of Computer Science and Engineering, City University, Khagan, \\ Ashulia, Dhaka-1216, Bangladesh \\ ${ }^{4}$ Associate Professor, Institute of Information Technology, Jahangirnagar University, \\ Savar, Dhaka - 1342, Bangladesh
}

\begin{abstract}
Underwater detector network is one amongst the foremost difficult and fascinating analysis arenas that open the door of pleasing plenty of researchers during this field of study. In several under water based sensor applications, nodes are square measured and through this the energy is affected. Thus, the mobility of each sensor nodes are measured through the water atmosphere from the water flow for sensor based protocol formations. Researchers have developed many routing protocols. However, those lost their charm with the time. This can be the demand of the age to supply associate degree upon energy-efficient and ascendable strong routing protocol for under water actuator networks. During this work, the authors tend to propose a customary routing protocol named level primarily based routing protocol (LBRP), reaching to offer strong, ascendable and energy economical routing. LBRP conjointly guarantees the most effective use of total energy consumption and ensures packet transmission which redirects as an additional reliability in compare to different routing protocols. In this work, the authors have used the level of forwarding node, residual energy and distance from the forwarding node to the causing node as a proof in multicasting technique comparisons. Throughout this work, the authors have got a recognition result concerning about $86.35 \%$ on the average in node multicasting performances. Simulation has been experienced each in a wheezy and quiet atmosphere which represents the endorsement of higher performance for the planned protocol.
\end{abstract}

\section{KEYWORDS}

UWSN; Better fitness; Multicasting; UWSN Applications

\section{INTRODUCTION}

Water is that the supply of human life. Quite the 70\% of the Earth's surface is roofed with water [17]. Water atmosphere observance, the management and protection of water resources are vital for our country to uncover the massive resources beneath water. Underwater sensing element Network (UWSN) permits real time observance of elect ocean areas with the 
supply of remote period of time wireless knowledge access. Variety of problems ought to be self-addressed whereas exploitation sensing element networks as an efficient technology for underwater systems [1]. An underwater network is usually created of several autonomous sensing element nodes that perform knowledge assortment operations moreover as store and forwarding operations to route the info that has been collected to a central node. Sensing element network technology is utilized extensively so as to watch the underwater atmosphere effectively and expeditiously. The underwater sensing element networks have some distinctive characteristics. It is low in value, little in size, low in power, multifunctional, and may simply communicate among short distances. Samples of sensors: Thermal, Visual, Light, Pressure, Temperature, Humidity etc.

The main challenges of deploying such a network [16] square measure is the restricted battery storage and computation, low information measure and high error rates. Errors square measure common in one, wireless communication. In a pair of, clamant measurements; three, node failure is expected that is quantifiable to an outsized variety of device nodes, and also survivability in harsh environments. Experiments depending on the nodes square measure is merely time and area intensive. In UWSNs, the node moves with the speed of 3-6 km/h [7] attributable to the water current. So, it is insufferable to progress routing protocols that work with the entire topology. Moreover, underwater device nodes cannot be recharged or modified attributable to the rough underwater surroundings. Underwater device nodes use associate modem whose propagation speed is $1500 \mathrm{~m} / \mathrm{s}$ [2] to transfer information to every different sensor. Our projected routing protocol is devised based mostly upon restricted battery and restricted information measure. This routing protocol provides shorter end-to-end delay with multicasting packet delivery in every receiving nodes through an optimized energy which is a remarkable change that signifies utmost benefits. This evading management of processing data packets for guiding the information packets to the destination entirely hoards up an outsized quantity of energy which is an optimized approach for maximum data transfer within the conjugate network nodes. Considering the noise in conjunction with that, the acceptable higher fitness of the nodes are calculated and measured accordingly. The illustration of our proposed network for the LBRP protocol is stated below:

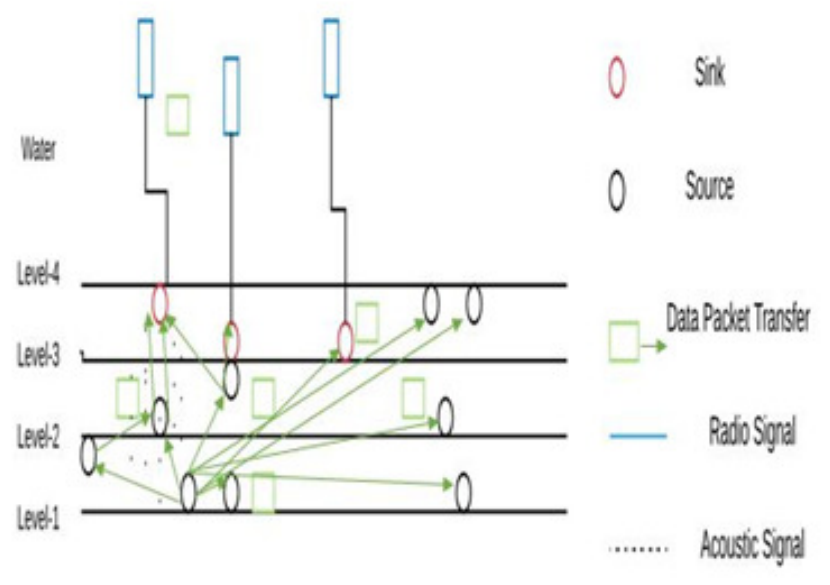

Figure 1: Proposed multicasting technique (LBRP) shows energy optimization scheme

Our key concern is to establish a routing protocol for UWSNs so that we can send data packet from source to destination efficiently meeting the challenges [14] of UWSNs. Our contribution to the work is listed below: 
1) Level Based Architecture

2) Localization free

3) Considering Noise Level in Link

4) Better Fitness calculation for Ease of Use

\section{RELATED WORKS}

One of the first topics for any network is routing, and routing protocols square measure thought to be an indictment of deciding and conserving the routes. Most of the analysis work touching on UWSNs are on the problems associated with the physical layer [16]. On the opposite hand, routing techniques square measure a relatively new arena of the network layer of UWSNs [13]. Thus, providing an economical routing algorithmic program becomes substantial. From figure-1 we will see the employment of underneath water device network in several surroundings [12].

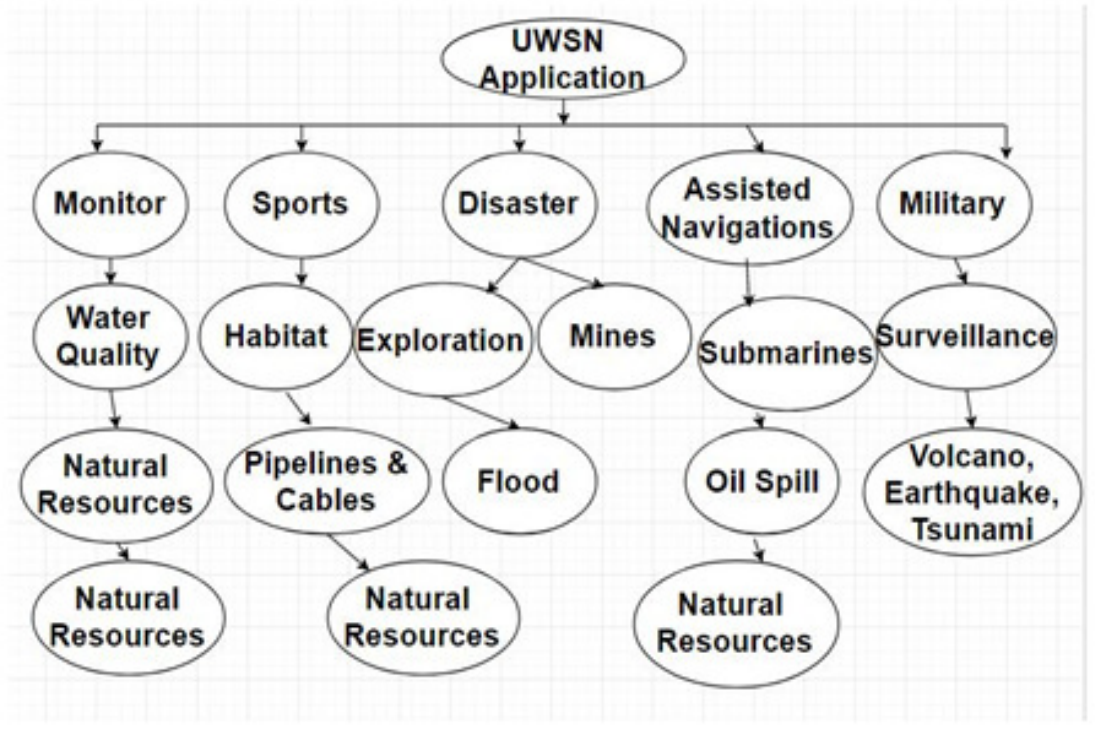

Figure 2: Classification of underwater wireless sensor network applications

Vector-based forwarding (VBF) [2] guides the packet from the supply to the destination. Packets are forwarded solely by those sensing element nodes that are at intervals then vary $\mathrm{R}$ of the vector. The forwarding method of VBF is assumed to be a routing pipe (virtual pipe) between the supply and therefore the destination nodes. The energy of the network is saved as a result of solely the nodes that bump into the forwarding path are concerned in packet routing. Nodes are mobile and are sensitive to the routing pipes radius. High communication time in dense networks is required and multiple nodes act as relay nodes. Some wide-area information assortment efforts are undertaken, however at quite coarse graininess (hundreds of sensors to hide the globe) [10]. Even once regional approaches are thought-about, they are typically wired and really high-priced [9]. 
During this paper, parabolic section is employed as a forwarding region for optimizing energy through parabola based routing (PBR) in Underwater sensing element Networks [3]. A replacement energy economical and depth primarily based routing protocol (EEDBR) for UWSNs is proposed in [4]; a replacement protocol within which solely the depth data of the nodes employed in the routing method. Also, to balance the general energy consumption and thereby increase the network time period, residual energy of the nodes thought-about within the routing calculations. The planned methodology not solely improves the general energy consumption of the network but the end-to-end delay is also considered and additionally reduced. All of those mentioned routing protocols for UWSNs are economical and effective in their own ways. In this paper, we have developed a routing protocol to beat the disadvantages of the vectorbased routing protocol [2] and (EEDBR) [4]. We have a tendency to introduce a customary technique by considering noise to assess the target node. LBRP improve the VBF, EEDBR in terms of the network time period, energy consumption, and end-to-end delay however, simply in distributed networks. Underwater nodes communicate with remote stations on the coast, that monitor and management these processes, victimization gateway nodes on the water surface as intermediaries [11] [12].

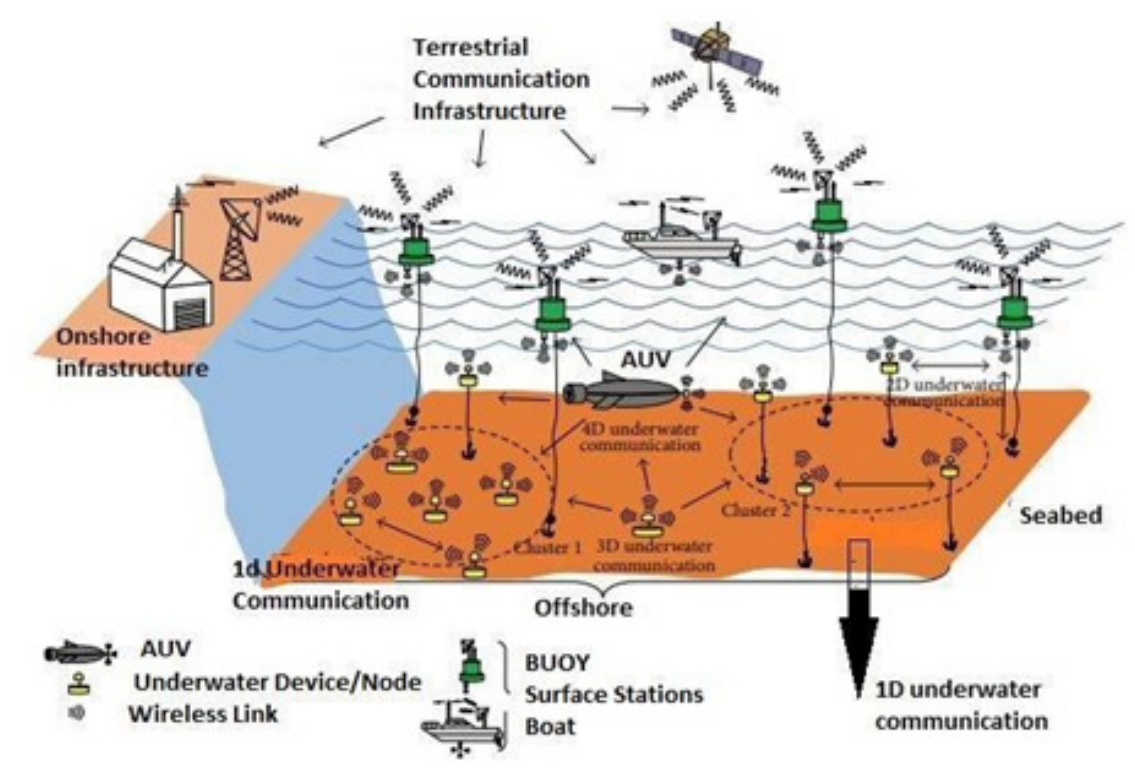

Figure 3: Underwater sensor network architecture

\section{Efficient Noise Aware LeVel Based Routing Protocol}

In this section, we've got mentioned specifications, Protocol summary and Protocol style. Finally, we have a tendency to show the algorithmic program and multicasting technique of the projected routing protocol.

\subsection{Network Architecture}

Efficient Noise Aware Level based Routing Protocol in UWSNs specifies and [19] Incredibly work out with a lot of help during this projected routing protocol. Assume that water depth is split in numerous levels as a result information delivery is far easier and economical. Associate example of such networks is incontestable in figure- 3 : 


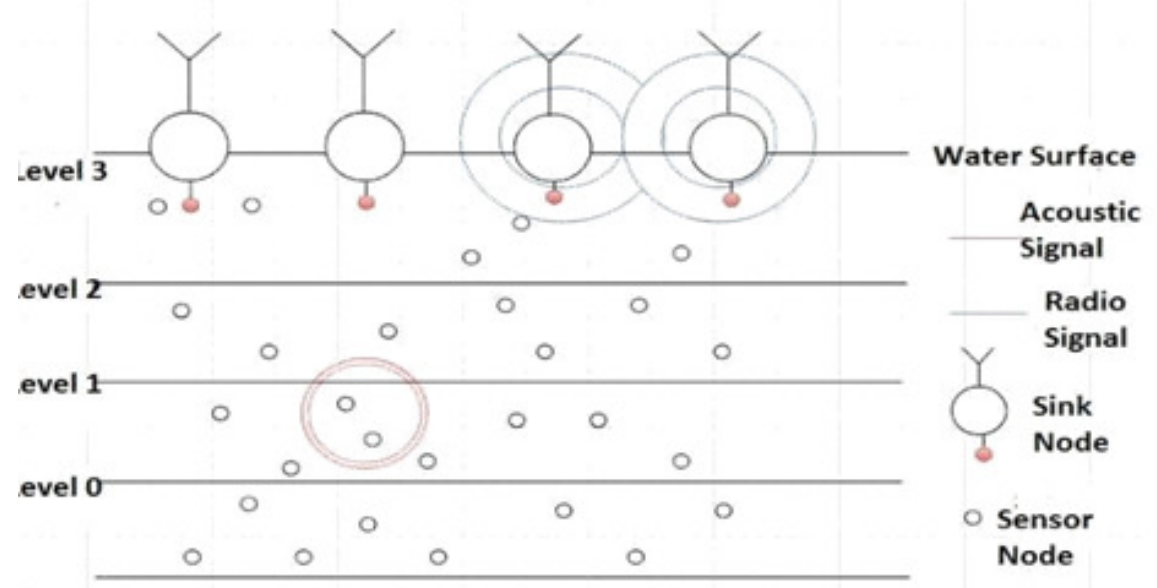

Figure 4: Noise Aware Level Based UWSNs architecture

Water depth is divided in different a level which is calculated in following way. Number of levels $=\frac{\text { Water depth }}{\text { level distence }}$

Where, Level distance is 300 meters.

In this Noise Aware Level primarily based network, there square measure multiple sink nodes out there in our planned specification that is mounted, wherever the forwarding node is often mobile. The water surface nodes that square measure referred to as sink nodes square measure equipped with the electronic equipment that's capable of capturing each radio-frequency and acoustic signals. The nodes that send and receive solely acoustic signals square measure deployed within the underwater surroundings. Underwater device nodes with acoustic modems square measure placed within the interested space and every such node is assumed seemingly to be an information supply. Underwater acoustic nodes will accumulate knowledge and conjointly assist to convey knowledge to the sinks. Once a sink node receives a packet from an underwater acoustic node, the sink node will converse with one another expeditiously via radio channels. The protocol tries to send a packet to any sink nodes on the surface as a result of if a surface node receives a packet it will send the packet alternative sinks or remote knowledge canters quickly thanks to the speed of radio- frequency (with a propagation speed of $3 \times 10^{8} \mathrm{~m} / \mathrm{s}$ in air) which is five orders of magnitudes higher than sound propagation (at the

speed of $1.5 \times 10^{3} \mathrm{~m} / \mathrm{s}$ in water) [7]. Here, the protocol does not concentrate to the communication between surface nodes. Instead, it tries to transmit a packet to any surface sinks and assumes that the packet reaches its destination [18]. The protocol has been designed by considering the very fact that each node is aware of its depth that is the vertical distance from the node's position to the surface and the position by itself.

\subsection{Overview of Efficient Noise Aware Level Based Routing Protocol for UWSNs}

The projected protocol consists of two parts named as Candidate Node choice part for shrewd Fitness by the causation Node and target node choice phase by the knowledge of routing Table and choose the forwarding node with highest fitness as Target Node [15]. Every of those elements are mentioned during this section. 


\subsection{Candidate Node Selection Phase}

- Sending detector Node broadcasts RREQ message at intervals higher one fourth portion of $\mathrm{R}$ circular vary.

- Receiving RREP message by causation Node containing link capability of the forwarding node whose level is above the causation Node.

- Calculating Fitness by the causation Node supported Link capability quantitative relation, Node Life time quantitative relation and Link Expiration Time.

\subsection{Target Node Selection Phase}

In this section, candidate Node choice section calculates fitness of causation node that is store in routing table. Once all higher fitness causation nodes square measure keep within the routing table and after victimization of the routing table, we tend to choose the best fitness causation node. The simplest fitness node is forwarded by victimization the multicasting method. Within the multicasting method, we tend to choose the forwarding node that is multicast to all or any nodes. We tend to then contemplate the minimum threshold fitness and most threshold fitness and calculate the common distinction between the utmost and minimum threshold fitness. Then we tend to once more contemplate the fitness of the forwarding node to calculate the common of the fitness distinction of these nodes from the utmost threshold and at last, we tend to calculate the amount of forwarding nodes that multicasts the node.

\subsection{Packet Format}

Two types of packets [6] area unit introduced during this protocol. First, the causing node broadcasts an effect packet named route request (RREQ) message to its adjacent node among the higher one fourth portion of R circular vary. So as to tell its neighbour of its location and depth, the route request message incorporates the causing node's ID and level of sender that area unit utilized by the causing node containing link capability of the forwarding node whose level is on top of the causing node. The fitness is calculated by the causing node being supported link capability quantitative relation, node life time quantitative relation and link expiration time. The packet format of RREQ is illustrated in Table 1.

Other management packets embody RREP message that carries the data of the forwarding node's link capability to forward the packet and ACK that is employed to verify the packet received by the forwarding node. The RREP message is illustrated in Table a pair of.

Table 1: Response Request (RREQ) Message

\section{\begin{tabular}{l|l} 
Sender ID & Level of Sender
\end{tabular}}

Table 2: Response Request (RREP) Message

\begin{tabular}{|c|c|}
\hline $\begin{array}{c}\text { Forwarding node } \\
\text { ID }\end{array}$ & Link Capacity \\
\hline
\end{tabular}




\subsection{Fitness Estimation of Proposed Protocol}

In this section, we have calculated fitness supported by the causing node. Our planned fitness estimation equation is illustrated in equation-1. First, we tend to calculate the link capability quantitative relation, node life time quantitative relation and link expiration time. Then we tend to place the link capability quantitative relation, node life time quantitative relation and link expiration time values in equation- 1 and calculated the fitness of the causing node.

$$
\begin{gathered}
\text { Fitness }\left(F T_{i}\right)=\text { Link Expiration Time }\left(L E T_{i}\right) \\
\times \frac{\text { Lifetime ratio }\left(L T^{r}\right)+\text { Link capacity ratio }\left(L C^{r}\right)}{1+a b s(\text { Link capactity ratio-life time ratio })} \ldots \ldots \ldots \ldots \ldots
\end{gathered}
$$

\subsection{Life Time Prediction Ratio $\left(L T^{r}\right)$}

In this section, we tend to calculate the life time prediction magnitude relation of the node. This is often the limit on the battery lifespan of a network that depends principally on tier one nodes. The time that a node's transceiver is active throughout one update amount is very important for battery life concerns. Every node uses a store and forward mechanism to forward a sequence of packets because it receives them so as to attenuate the active time of its transceiver. Taking under consideration collisions and retransmissions [5], the full active time for a tier one transceiver in one update amount is:

$$
\text { Total Time }\left({ }^{\text {Ttotal }}\right)=1.23(N \max +N \max / 5)
$$

Where, Nmax defines the most range of packets in seconds. Consequent step is choosing an influence supply. We tend to contemplate that we have got three off-the shelf $9 \mathrm{~V}, 1.2$ Amp-hour batteries at every node. The overall energy obtainable at every node is: $E_{*}=3 \times 9 \times 1.2=32.4$ in V.A hour. The overall active time of a transceiver is thus the magnitude relation of the overall energy to the ability consumed in one frame:

$$
\text { Total active time }(\text { Tactive })=\frac{E t}{\text { Pframe }}=\frac{32.4}{\text { Pframe }}
$$

in hours. A node's transceiver is simply active for a fraction of the time in every update amount of $\mathrm{R}$ seconds. Therefore, the battery period of time of a node is expressed by:

$$
\text { Life time of a node }\left(L T_{i}\right)=\frac{\text { Tactive }}{T_{\text {total }}} \frac{R}{\times 24}
$$

in days, where $\mathrm{R}$ is in seconds.

\subsection{Link Capacity Ratio $\left(L C^{r}\right)$}

In this section, we calculated the link capacity ratio of the node by using this equation:

$$
\text { Link capacity of a reciver }\left(L C_{i}\right)=B \times \log _{2}\left(1+S N R_{i}\right)
$$

Where, B is the bandwidth of the channel and $S N R_{i}$ is the signal-to-noise ratio of the link between the sending node and the forwarding node $f_{\text {: }}$.

The passive sonar equation [6] indicates that the signal-to-noise ratio (SNR) per bit $\gamma_{h}$ of an emitted underwater signal at the receiver is 


$$
\gamma_{b}=S L-T L-N L+D I
$$

Where, $S L$ is the source level, $T L$ the transmission loss, $N L$ the noise level and $D I$ the directivity index. The unit of all quantities is $\mathrm{dB}$. Since unidirectional hydrophones are used, DI is 0 .

\subsection{Link Expiration Time $\left(L E T_{i}\right)$}

Link [18] stability of any twoRnodes means the duration of the connectivity of the two nodes within a fixed range Let $n_{1}$ and $n$, be two nodes within a fixed range $R$. These two nodes move $\theta_{1}, \Phi_{1}$ and $\theta_{2}$, direction in three-dimensional space of underwater respectively. Let their initial position be $, v_{1}^{\prime}, z_{1}^{\prime}$, and $x_{\sim}^{\prime}, v_{\sim}^{\prime}, z_{\sim}^{\prime}$ respectively after time $t$ their new coordinates will be $x_{1}, y_{1}$, and $x_{2}, y_{2}, z_{2} \underset{x_{1}^{\prime}}{\Phi_{1}^{\prime}}$ respectively. Suppose they travel at the speed of $v_{1} \mathrm{~m} / \mathrm{s}$ and $v_{2} \mathrm{~m} / \mathrm{s}$ respectively and after time $t n_{1}$ passes $d_{1}$ meter and $n$, passes $d_{3}$ meter [7].

$$
d_{1}=v_{1} t \text { and } d_{2}=v_{2} t
$$

The distance between the two nodes at time and $d_{2}=$ can be found as follows:

$$
\begin{aligned}
& \quad a=\left(x_{1}^{\prime}-x_{2}^{\prime}\right), \quad b=\left(y_{1}^{\prime}-y_{2}^{\prime}\right) \text { and } c=\left(z_{1}^{\prime}-z_{2}^{\prime}\right) \\
& e=\left(v_{1} \sin \theta_{1} \cos \Phi_{1}-\sin \theta_{2} \cos \Phi_{2}\right) \\
& f=\left(v_{1} \sin \theta_{1} \sin \Phi_{1}-\sin \theta_{2} \sin \Phi_{2}\right) \\
& g=\left(v_{1} \cos \theta_{1}-v_{1} \cos \theta_{2}\right)
\end{aligned}
$$

Now, the distance between the two nodes after time

can be calculated as follows:

$D^{2}=(a+e t)^{2}+(b+f t)^{2}+(c+g t)^{2},\left(t^{2}+f^{2}+g^{2}\right)+t(2 a e+2 b f+2 c f) a^{2}$ 


\subsection{The Routing Algorithms of the Proposed Routing Protocol}

Our proposed routing protocol algorithm for the Routing Table formation is illustrated in Algorithm-1. Here, $n_{n}$ level of the sending node and $f_{m}$ level of the forwarding node and $F t_{i}$ is the calculated fitness.

\section{Algorithm-1}

Require: Control Packet: RREQ, RREP, Data

Packet

Ensure: Forwarding Data Packet

Broadcast RREQ message by the sending ns

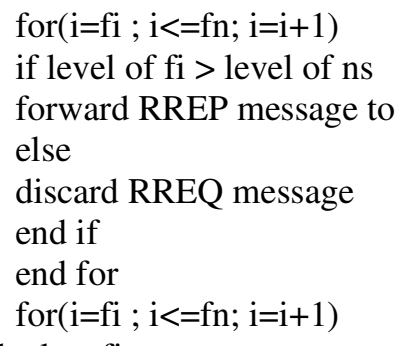

\subsection{Multicasting Technique}

In this section, we have a tendency to

use the multicasting technique for forwarding the node. We have selected the simplest fitness of node from the routing table and multicast this node.

- Selection of Forwarding Nodes

- let maximum threshold fitness $F_{t h}^{\max }$ and minimum threshold fitness $F_{t h}^{\min }$

- let the average difference between $F_{t h}^{\max }$ and $F_{t h}^{\min }$ be $r_{a v g}^{\max }$

- let fitness of forwarding nodes be $F 1, F 2, \ldots \ldots F n$ and the average of the fitness difference of those node from maximum threshold fitness $F_{t h}^{\max }$ is

$$
r_{a v g}=\frac{\sum_{i=1}^{n}\left(F_{t h}^{\max }-F i\right)}{n}
$$

Then number of Forwarding Node

$$
n=1+\text { ceiling }\left[\frac{N \times \text { padd }}{100}\right]
$$

Where, padd $=\frac{p r}{r_{\text {avg }}^{\max }} \times r_{\text {avg }}$

$p r$ is the percentage of additional forwarding node. 


\section{RESUlt AND ANALYSIS}

In this section, the result and therefore the analysis of the simulation area unit mentioned well. The simulation of LBRP, EEDBR [4] and VBF [3] area unit are explained below.

\subsection{Network life Time}

The comparison of the performances of LBRP, EEDBR [4] and VBF [2] in terms of network lifetime is illustrated in Figure 5

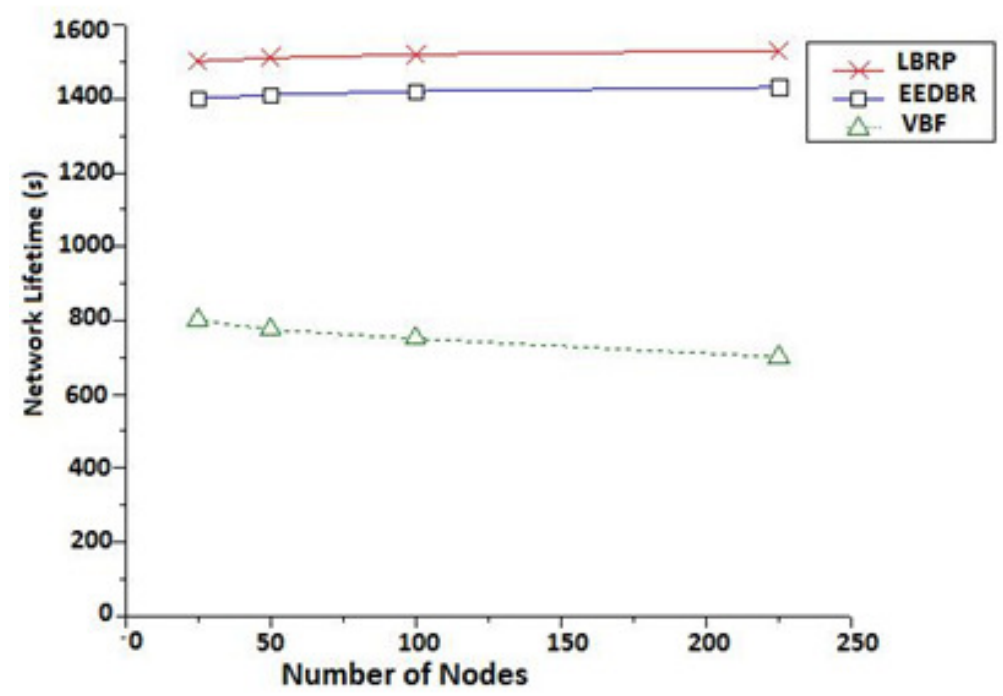

Figure 5: Comparison of network lifetime of different Level Based Routing Protocol (LBRPs)

It is determined that LBRP offers improved performance over EEDBR within the perspective of network [20] period of time. LBRP exceeds the network period of time of VBF, EEDBR as a result of VBF invariably chooses the nodes within an exceedingly mounted vector, and as a result, a sensing element node [21] is also selected once more and once more to forward knowledge. Consequently, the energy of such nodes is exhausted quickly, and the period of time of these nodes expires shortly. The chosen EEDBR forwarding node is predicated solely on depth. Therefore, any node features a probably likelihood to die shortly. EEDBR employs the energy reconciliation among the sensing element nodes, thus EEDBR desires a lot of energy. On the opposite hand, LBRP does not forward knowledge supported depth, it divides water depth in numerous levels. Packets are forwarded in higher levels and nodes are sent containing link capability of the forwarding node whose level is over the causing node [22]. As LBRP uses the multicasting technique to multicast the higher fitness to alternative nodes, knowledge loss is lowest and needs less energy, resulting in extended battery period of time.

\subsection{Total Energy Consumption}

The comparison of the performances of LBRP, EEDBR [4] and VBF [2] in terms of energy consumption is illustrated in Figure 6.

It is seen that the planned protocol consumes less energy than that of the VBF, EEDBR protocol. Since level wise divisions of observance setting reduces the energy consumption, no further 
electronic equipment is required within the detector node to live the depth below the water. In VBF, over one node attends in forwarding a similar packet [23]; thus higher energy is consumed in VBF protocol. In VBF, node quality is not guarded; thus with the rise of nodes and node quality, additional energy is being consumed. The energy consumption in EEDBR is more than LBRP. As a result of in EEDBR, additional nodes involve in forwarding similar packets because it might happen that over one node has a similar depth and it causes a similar holding time.

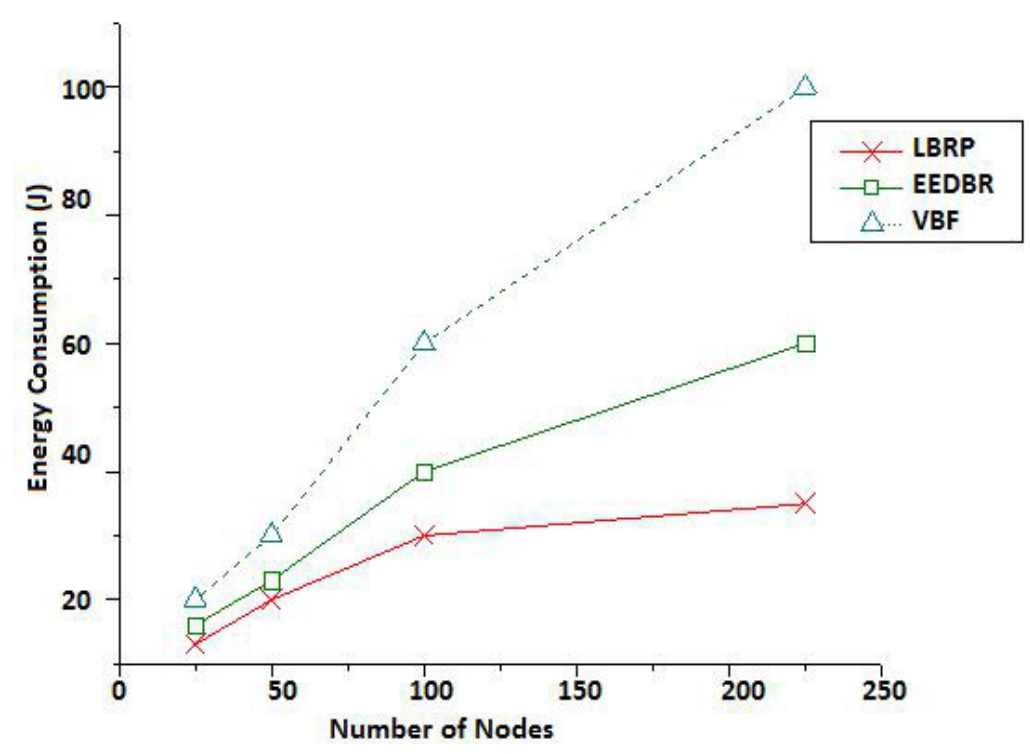

Figure 6: Comparison of energy consumption of different nodes

\subsection{Average End-to-End Delay}

The comparison of the performances of LBRP, EEDBR [4] and VBF [2] in terms of Average End-to-End Delay is illustrated in Figure 7.

In VBF, it takes longer to find the destination node and for the response to come back from the destination node in VBF, that the average end-to-end delay in VBF is larger. For VBF to handle node quality, no direct technique is applied. As a result, less range of the forwarding nodes attend in forwarding packet with increasing node quality, and it will increase the end-to-end delay. In EEDBR, each detector node holds the packet for an explicit time proportional to the depth of the detector node. Therefore, EEDBR includes a long finish end-to-end delay. In LBRP packet delivery quantitative relation is healthier than VBF, EEDBR. As a result of the routing table selects higher fitness of forwarding node, the node uses multicasting technique to multicast the higher fitness to a different node. So, packet delivery quantitative relation is accumulated. 


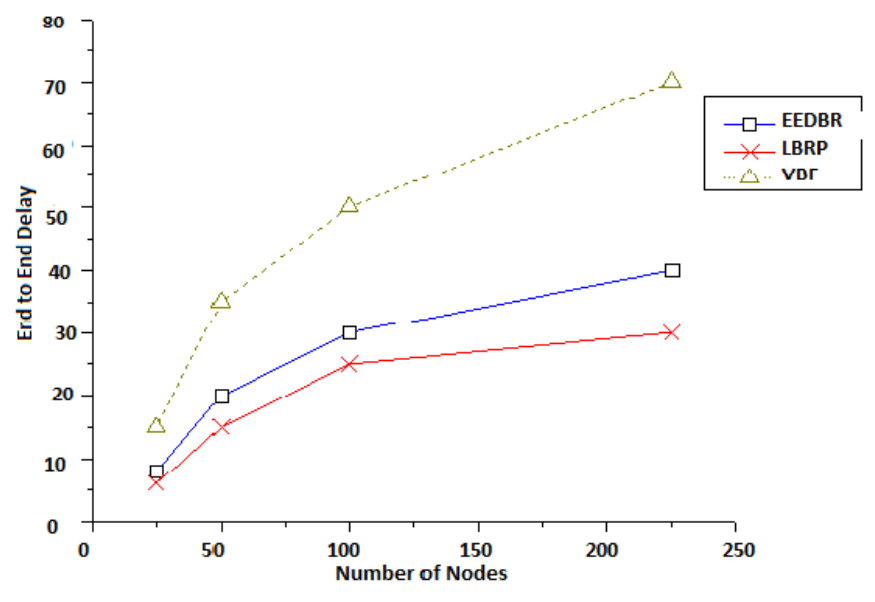

Figure 7: Comparison of end-to-end delay of different nodes

\subsection{Packet Delivery Ratio}

The comparison of the performances of LBRP, EEDBR [4] and VBF [2] in terms of packet delivery quantitative relation is illustrated in Figure 8.

In VBF, solely those nodes that square measure within the vector participate in forwarding packet. With the increasing node quality, the node will be out of the vector, and this result reduces the packet delivery quantitative relation in VBF. In EEDBR routing protocol depends on depth and distributed energy of all nodes. So, the packet has very little result and reduces the packet delivery quantitative relation in EEDBR. Contrarily, LBRP has abundant less end-to-end delay by comparison the VBF, EEDBR. LBRP perpetually tries to send packets to higher levels wherever the forwarding [25] node is beyond causing node, and calculates higher fitness supported link capability between the forwarding and therefore the causing node. Contrary to LBRP, because the speed of water current will increase, the end-to-end delay decreases as in the planned protocol.

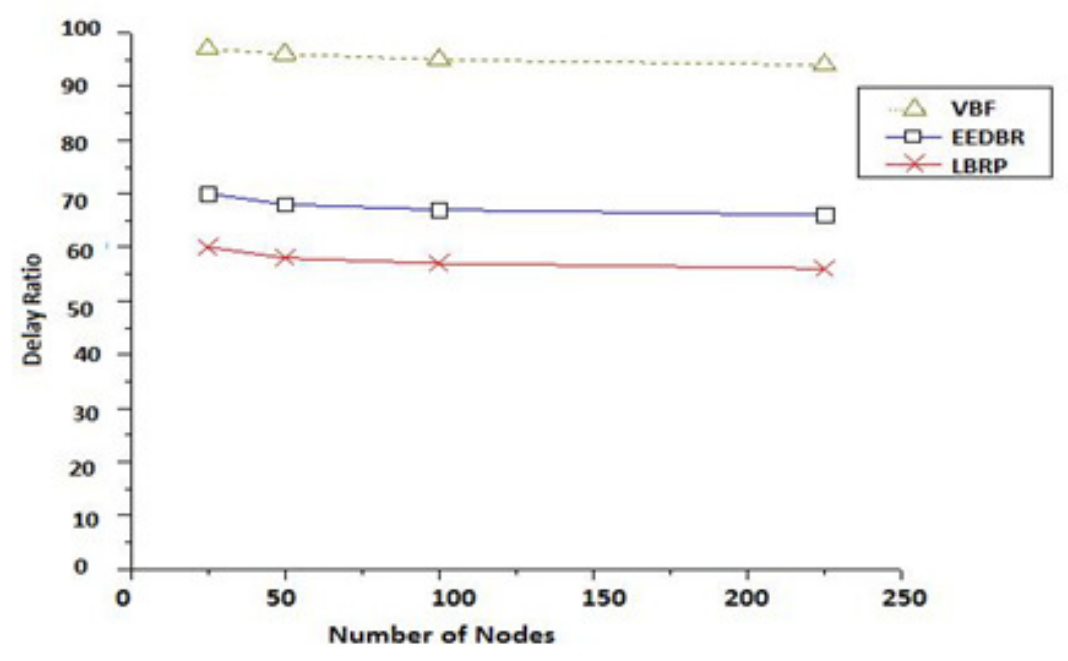

Figure 8: Comparison of packet delivery ratio of different sensor node 


\section{Simulation ENVIRONMENT}

Matlab is used to simulate this experiment. In some cases codeblocks ver.13.2 was used for getting the output and used it onto matlab for improving overall performance.

\section{Conclusion}

The protocol performs better in terms of energy consumption and ensures that the link is less noisy. Level-wise divisions of monitoring environment reduce the energy consumption because no extra circuitry is needed in the sensor node to measure the depth under the water.

\section{ACKNOWLEDGEMENTS}

This work is supported by my colleague Milon Biswas and his friend Md.Julkar Nayeen Mahi. Both of them are Lecturer in Dept. of Computer Science and Engineering, Bangladesh University of Business and Technology (BUBT) and City University, Khagan, Ashulia; Bangladesh respectfully. The author is grateful for their support. The author also give thanks to Dr.Md.Whaiduzzaman, Associate Professor, Institute of Information Technology (IIT), Jahangirnagar University, Dhaka; Bangladesh for his kind supervision in this whole work.

\section{REFERENCES}

[1] Akyildiz IF, Pompili D, Melodia T. Underwater acoustic sensor networks: research challenges. Ad hoc networks. 2005 May 1;3(3):257-79.

[2] Xie P, Cui JH, Lao L. VBF: vector-based forwarding protocol for underwater sensor networks. InInternational conference on research in networking 2006 May 15 (pp. 1216-1221). Springer, Berlin, Heidelberg.

[3] Dhurandher SK, Obaidat MS, Goel S, Gupta A. Optimizing energy through parabola based routing in underwater sensor networks. InGlobal Telecommunications Conference (GLOBECOM 2011), 2011 IEEE 2011 Dec 5 (pp. 1-5). IEEE.

[4] Zenia NZ, Aseeri M, Ahmed MR, Chowdhury ZI, Kaiser MS. Energy-efficiency and reliability in MAC and routing protocols for underwater wireless sensor network: A survey. Journal of Network and Computer Applications. 2016 Aug 1;71:72-85.

[5] Rahman MT, Mahi MJ, Biswas M, Kaiser MS, Al Mamun S. Performance evaluation of a portable PABX system through developing new bandwidth optimization technique. InElectrical Engineering and Information Communication Technology (ICEEICT), 2015 International Conference on 2015 May 21 (pp. 1-5). IEEE.

[6] Jurdak R, Lopes CV, Baldi P. Battery lifetime estimation and optimization for underwater sensor networks. IEEE Sensor Network Operations. 2004 Jun;2006:397-420.

[7] Uddin M. Link expiration time-aware routing protocol for UWSNs. Journal of Sensors. 2013;2013.

[8] Heidemann J, Ye W, Wills J, Syed A, Li Y. Research challenges and applications for underwater sensor networking. InWireless Communications and Networking Conference, 2006. WCNC 2006. IEEE 2006 Apr 3 (Vol. 1, pp. 228-235). IEEE.

[9] Felemban E. Advanced border intrusion detection and surveillance using wireless sensor network technology. International Journal of Communications, Network and System Sciences. 2013 May $15 ; 6(05): 251$. 
[10] Felemban E, Shaikh FK, Qureshi UM, Sheikh AA, Qaisar SB. Underwater sensor network applications: A comprehensive survey. International Journal of Distributed Sensor Networks. 2015 Nov 1;11(11):896832.

[11] Menon KU, Divya P, Ramesh MV. Wireless sensor network for river water quality monitoring in India. InComputing Communication \& Networking Technologies (ICCCNT), 2012 Third International Conference on 2012 Jul 26 (pp. 1-7). IEEE.

[12] Faustine A, Mvuma AN, Mongi HJ, Gabriel MC, Tenge AJ, Kucel SB. Wireless sensor networks for water quality monitoring and control within lake victoria basin: prototype development. Wireless Sensor Network. 2014 Dec 4;6(12):281.

[13] Shakir M, Khan MA, Malik SA. Izhar-ul-Haq,-Design of underwater sensor networks for water quality monitoring. World Applied Sciences Journal. 2012;17(11):1441-4.

[14] Kumar G, Rai MK, Saha R, Kim HJ. An improved DV-Hop localization with minimum connected dominating set for mobile nodes in wireless sensor networks. International Journal of Distributed Sensor Networks. 2018 Jan;14(1):1550147718755636.

[15] Muhammed D, Anisi MH, Zareei M, Vargas-Rosales C, Khan A. Game Theory-Based Cooperation for Underwater Acoustic Sensor Networks: Taxonomy, Review, Research Challenges and Directions. Sensors. 2018 Feb 1;18(2):425.

[16] Cayirci E, Tezcan H, Dogan Y, Coskun V. Wireless sensor networks for underwater surveillance systems. Ad Hoc Networks. 2006 Jul 1;4(4):431-46.

[17] Mohamed N, Jawhar I, Al-Jaroodi J, Zhang L. Sensor network architectures for monitoring underwater pipelines. Sensors. 2011 Nov 15;11(11):10738-64.

[18] Mahi MJ, Rahad KA, Biswas M, Islam R, Chowdhury ZI. An Accident Detection System for a Single VANET at Low Cost Module. IEEE, TechSym. 2016 Sep 17;4(1):44-45,

[19] Bhuiyan KA, Whaiduzzaman M, Nasir MK. Efficiency and Performance analysis of routing protocols in WSN. International Journal of Advanced Engineering Research and Science.;4(4).

[20] Yahya A, ul Islam S, Akhunzada A, Ahmed G, Shamshirband S, Lloret J. Towards Efficient Sink Mobility in Underwater Wireless Sensor Networks. Energies. 2018;11(6):1-2.

[21] Lloret J. Underwater sensor nodes and networks. 2013: 11782-11796

[22] Ahmed M, Salleh M, Channa MI. Routing protocols based on node mobility for underwater wireless sensor network (UWSN): a survey. Journal of Network and Computer Applications. 2017 Jan $15 ; 78: 242-52$.

[23] Goyal N, Dave M, Verma AK. Data aggregation in underwater wireless sensor network: Recent approaches and issues. Journal of King Saud University-Computer and Information Sciences. 2017 May 2.

[24] Sahana S, Singh K, Kumar R, Das S. A review of underwater wireless sensor network routing protocols and challenges. InNext-Generation Networks 2018 (pp. 505-512). Springer, Singapore.

[25] Bansal R, Maheshwari S, Awwal P. Challenges and Issues in Implementation of Underwater Wireless Sensor Networks. InOptical and Wireless Technologies 2018 (pp. 507-514). Springer, Singapore. 


\section{AUTHORS}

Md. Ashraful Islam has a Bachelor's Degree in Computer Science and Engineering from Bangladesh University of Business and Technology, BUBT. He has recently presented his paper on Mathematical Induction at the ICMEAS Conference which was organized by MIST and BUET. His research areas include Algorithms, Big Data, Image Processing, Data Mining, Cyber Security, and Networking. He is currently working as a lecturer at BUBT, and was a former Lecturer at Varendra University

Milon Biswas has successfully completed his B.Sc. degree in IT, Jahangirnagar University, Bangladesh and currently studying in M.Sc. at the same institute. He has one conference paper at IEEE engineering community. His current research interests are mainly in Computer networks, Image processing and Cloud computing.
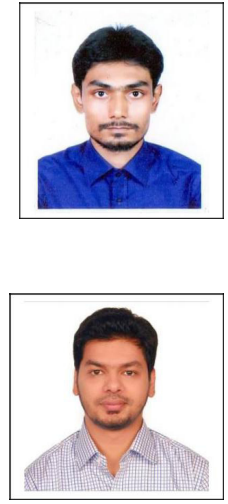

Md.Julkar Nayeen Mahi has successfully completed his B.Sc. degree in IT, Jahangirnagar University, Bangladesh and currently studying in M.Sc. at the same institute. Currently he is serving as a 'Lecturer' in City University, Bangladesh. His current research interests are mainly in Distributed computer networks, Embedded systems, IoT, Data mining, Cloud computing, Operating Systems Scheduling approach, Wireless Sensor Networks.

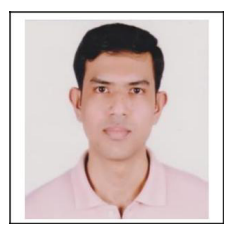

Md Whaiduzzaman completed his undergraduate degree in Electronics and Computer Science with first class from Jahangirnagar University and he obtained M.Sc. degree in Telecommunication and Computer Network Engineering from London, South Bank University, UK. He serves as an Associate Professor in the Institute of Information Technology (IIT), Jahangirnagar University, Bangladesh. His research interests are Mobile Cloud Computing, Vehicular cloud computing, Fog Computing, Mobile and

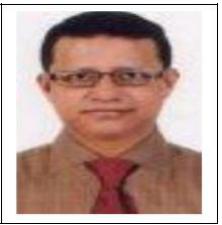
wireless communications and security issues. 